\title{
O.N THE SUBPLLSE DRIFT IN PSR 2303+30
}

\author{
J. A. GIL \\ The Aitrofomical Centre of Zielona Góra and Institute of Physics, Pedagogical University \\ T. H. HANKINS \\ Very Large Array, National Radio Astronomy Observatory \\ and Department of Physics, New Mexico Institute of Mining and Technology \\ L. Nowakowski \\ Department of Physics, Lniversity of Puerto Rico at Mayaguez
}

\begin{abstract}
We present an analysis of high signal-to-noise ratio, $430-\mathrm{MHz}$ totai intensity single pulse observations from PSR 2303+30 recorded at the Arecibo Observatory in August 1986. We believe that the regular subpulse modulation observed in this pulsar can be explained only in terms of subpulseassociated emission beams rotating around the axis of the pulsar beam. This idea was first introduced by Ruderman and Sutherland (1975) in their spark noodel. We use the quantitative prediction of this model to calculate the pulsar magnetic field. For this reason we need to estimate the rotation period of a spark around the magnetic pole. We estimate this period by reproducing the observed pulse sequences within an angular beaming model of subpulse emission and fitting this model to the data.
\end{abstract}

\section{Observations}

Power spectra of PSR $2303+30$ show a strong peak close to the Nyquist frequency (e.g., Sieber and Oster 1975), associated with so called even-odd modulation. Two typical sequences of individual pulses are presented in figures $1 \mathrm{a}$ and $1 \mathrm{~b}$. The apparent drift bands visible in these figures correspond to a phenomenon which we will call the secondary drift. These bands are formed from even and odd pulses separately. Individual pulses alternate between single- and double-subpulse forms with astonishing precision. The former usually has a strong central subpulse, while the latter has two weaker subpulses. The primary subpulse drift rate of $\sim 2.5^{\circ} / P$ is very fast in this $P=1.57$-s period pulsar, resulting in a subpulse drift across the entire pulse window during one pulsar period.

One can distinguish three different types of modulation in sequences presented in figures 1a and 1b. Type $A$, visible in the lower part of figure 1a, demonstrates constant phase and intensity in both single and double pulses. Type $A$ is preceded and followed in figure la by Type $B$ in which both the phase and intensity of subpulses are modulated along secondary drift bands. The modulation is very precise with the subpulse intensity strongly depending on its phase. The subpulses at the trailing edge are weak; the intensity gradually increases until it reaches a maximum near the center of the pulse window, and then it gradually decreases again toward the leading edge. Type $C$, which we will not discuss in this paper, is erratic and noisy. The extremely regular modulations visible in figure $1 \mathrm{a}$ and $1 \mathrm{~b}$ can be explained by rotation of angular beams associated with individual subpulses around the pulsar beam axis. Type $A$ corresponds to a velocity of rotation $v=360^{\circ} /(2 n P)$, where $n$ is a total number of subpulse beams within the instant pulsar beam and type $B$ corresponds to a velocity slightly different from that of type $A$. In principle, the drift velocity $v$ depends on a number $n$ of subpulse beams. In this case, it is obvious from visual inspection of figure $1 \mathrm{a}$ and $1 \mathrm{~b}$ that $n$ is at least 3 . We will argue in the next section that the actual number of subpulse beams is 4 . This means that $v=45^{\circ}$ per period and a subpulse beam completes one revolution around the magnetic axis in about $360^{\circ} / 45^{\circ}=8 P$. In modulation of type $B$ of which a long sequence is presented in figure $1 \mathrm{~b}$, the velocity of rotation slightly differs from $45^{\circ} / P$. This explains the strong dependence of the subpulse intensity on its phase within the pulse window.

\section{A model}

We have simulated the secondary drift using a scheme developed by Gil (1984). A number $n$ of sparks (Ruderman and Sutherland, 1975) rotate on the surface of polar cap with radius $r_{\mathrm{p}}$ given by Goldreich and Julian (1969). These sparks deliver $e^{+} e^{-}$plasma into the magnetosphere along dipolar field lines. At some distance $r$ from the star a plasma column associated with the spark radiates a subpulse beam which rotates around the magnetic axis. We have assumed that radiation is strongly 

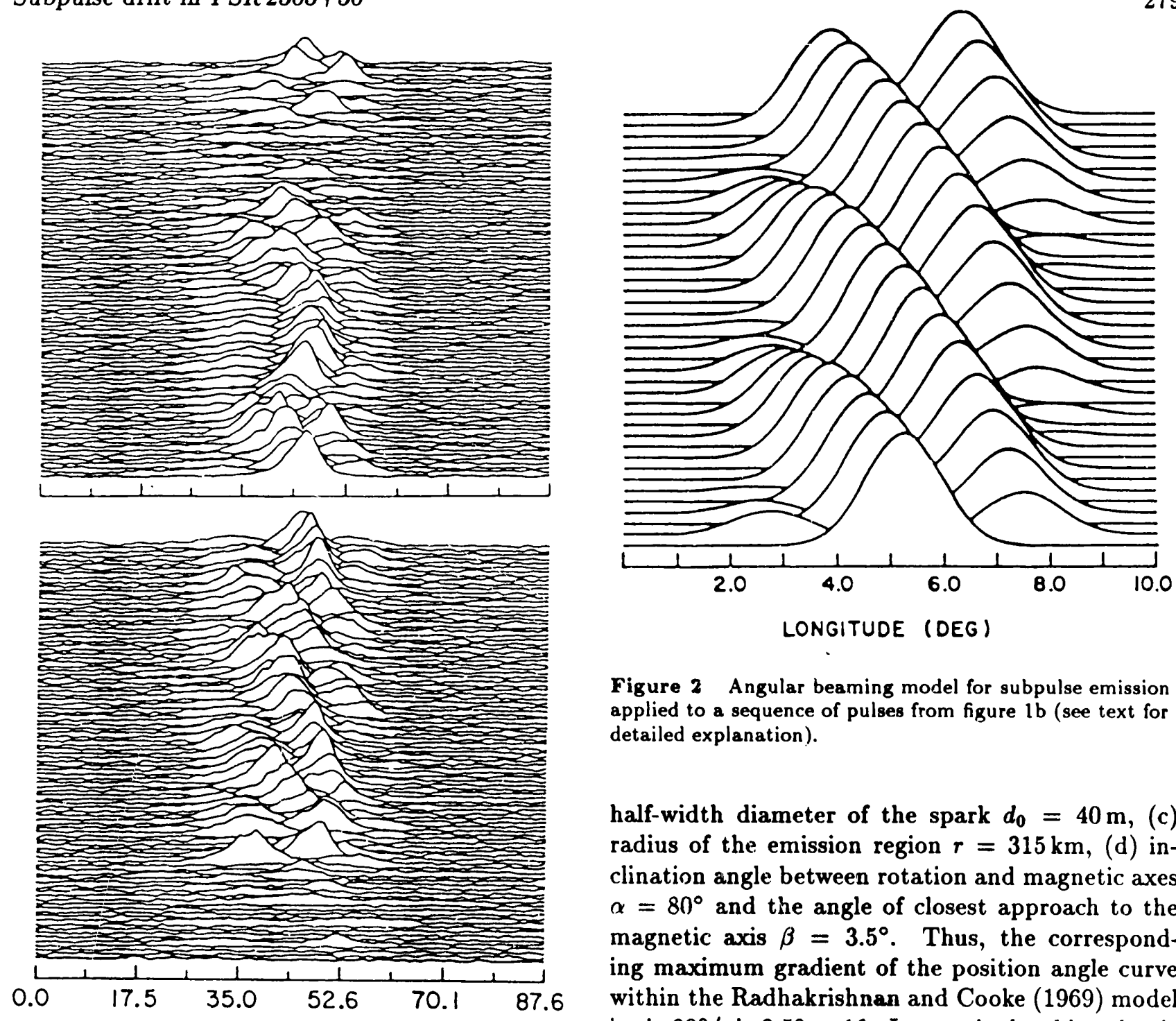

LONGITUDE (DEG)

Figure 2 Angular beaming model for subpulse emission applied to a sequence of pulses from figure $1 b$ (see text for detailed explanation).

half-width diameter of the spark $d_{0}=40 \mathrm{~m}$, (c) radius of the emission region $r=315 \mathrm{~km}$, (d) inclination angle between rotation and magnetic axes $\alpha=80^{\circ}$ and the angle of closest approach to the magnetic axis $\beta=3.5^{\circ}$. Thus, the corresponding maximum gradient of the position angle curve within the Radhakrishnan and Cooke (1969) model is $\sin 80^{\circ} / \sin 3.5^{\circ}=16$. Interestingly, this value is very consistent with the curve presented in figure 5 of Gil (1992). The simulations performed suggest that the observational data presented in figures 1a and $1 \mathrm{~b}$ can be reproduced only with parameter values close to the set described above.

Within the Ruderman and Sutherland (1975)

beamed tangent to dipolar field lines. The energy distribution within a beam is assumed to be Gaussian with parameters determined from the first subpulse in figure 1a.

We have attempted to fit precisely data from figure $1 \mathrm{~b}$ with the above model. The task was not trivial because one has to fit several parameters at the same time: subpulse width, subpulse spacing, pulse window width, secondary drift-band slope and the form of intensity modulation along a drift band. Therefore, the fitting procedure unambiguously determines the number of sparks $n=4$. These sparks were separated by about $90^{\circ}$ from each other. The results of the simulation are presented in figure 2. The adopted drift velocity is $v=48^{\circ} / P$. The geometrical parameters of the simulation are: (a) distance from the magnetic axis to the center of the spark $d=70 \mathrm{~m}$ (polar cap radius $r_{\mathrm{p}}=115 \mathrm{~m}$ ), (b) model (see also Filippenko and Radhakrishnan 1982), the rotation of sparks around the pole is caused by the so-called $E \times B$ drift. A pulsar magnetic field at the pole can be calculated from timing data $\sin \alpha B=3.2 \times 10^{19}(P \dot{P})^{1 / 2}$ Gauss. For PSR 2303+30 $\dot{P}=2.89 \times 10^{-15}$ and $B_{12}=$ $2.2 / \sin \alpha$, where $B_{12}=B / 10^{12}$. The magnetic field can be measured independently within the Ruderman and Sutherland model if one knows the time $\hat{P}$ for a spark to complete one rotation around the magnetic axis $B_{12}=\hat{P} P / 5.6$. Fron the above simulations $\hat{P}=360^{\circ} v=8 P$ and therefore $B_{12}=$ $8 P^{2} / 5.6=3.3$ in excellent agreement with the timing value of $B_{12}=2.2 / \sin \alpha$.

Acknowledgment: The Arecibo Observatory is operated by Cornell University under a management agreement with the National Science Foundation. 